\title{
Recognition of Direction of New Apertures from the Elongated Speckle Images: Simulation
}

\author{
Abdallah Mohamed Hamed \\ Physics Department, Faculty of Science, Ain Shams University, Cairo, Egypt \\ Email: Amhamed73@hotmail.com \\ Received January 31, 2013; revised March 2, 2013; accepted March 9, 2013
}

Copyright (C) 2013 Abdallah Mohamed Hamed. This is an open access article distributed under the Creative Commons Attribution License, which permits unrestricted use, distribution, and reproduction in any medium, provided the original work is properly cited.

\begin{abstract}
In this paper, we present an elongated speckle images produced from diffusers using sharp elliptical apertures. The orientation of the elliptic aperture is recognized from the direction of the elongation in the speckle images. The aperture tilting out of the plane is investigated. Three models of elliptical apertures are considered and the corresponding speckle images are obtained. The 1st model is composed of two orthogonal ellipses or plus symbol pupil; the 2nd has four symmetric ellipses with an angle of $45^{\circ}$ between each of them or in the form of a snow flake pupil and the 3rd model looks like an airplane. Also, the autocorrelation profiles of the speckle images corresponding to the diffused airplane are obtained from which the average speckle size is computed. Finally, the reconstructed images of the described elliptical models and its autocorrelation images, making use of Mat lab code, are obtained.
\end{abstract}

Keywords: Digital Imaging; Sharp Elliptical Apertures; Plus Symbol and Snow Flake Pupils

\section{Introduction}

The production of elongated speckle images was obtained using the mechanical scanning of the static speckle pattern [1]. The author presented a technique of spatially oriented speckle-screen encoding to improve the grating encoding technique for white-light image processing. Also, an artificial screen composed of small strips photographed several times on a high resolution film is designed to obtain elongation of nearly ten times the average grain size of natural speckle [2]. In a recent publication by the author [3], numerical elliptical apertures of small elliptic shapes are analyzed and the Fourier transform is operated giving the speckle images of diffusers modulated by these elliptic apertures.

An approach for determining the roughness of engineering surfaces is resulted from the speckle elongation effect. The laser speckle pattern, arising from light scattered from rough surfaces that are illuminated by polychromatic laser light, is detected in the far-field region. The incoherent superposition of these light intensities and the angular dispersion cause the effect of speckle elongation $[4,5]$. This is characterized by increasing speckle widths and leads to a radial structure of the speckle patterns. With increasing surface roughness, the elongation is replaced more and more by the de-correlation of the monochromatic speckle patterns for the different wavelengths. Such effects were detected with the CCD technique and analyzed by local autocorrelation functions of intensity fluctuations that were calculated for different areas of the speckle patterns. Hence, the autocorrelation method is applied to process laser speckle patterns. The relation between surface roughness, speckle elongation, and correlation length of autocorrelation function can be obtained. Consequently, the measured surface roughness can be achieved [6]. An oriented photographic diffuser was used to record an elongated speckle pattern. It is found that the contrast transfer, when gratings are imaged through the slits in the diffuser, is considerably higher compared to imaging through a circular pinhole of comparable dimensions [7]. An autocorrelation algorithm for speckle size evaluation has been investigated [8-11]. The authors measured the average speckle size from the auto-covariance function of the digitized intensity speckle pattern. The spatial characteristics such as speckle size can be used to measure the roughness of surfaces $[12,13]$. An important remark must be taken into consideration during the recording of speckle data which states that the speckle size must be greater than the pixel size of the CCD camera in order to resolve variations in speckle intensity [14]. Recently, a Fast calculation method for optical diffraction on tilted plane was investigated [15]. 
In this work, two sharp elliptical apertures with different orientations are proposed and the corresponding elongated speckle images are obtained. Also, three different new models in the form of the plus symbol, a snowflake and an airplane, are numerically simulated and the corresponding speckle images are computed and plotted. Finally, the reconstructed images of the models and the autocorrelation of these models are plotted. It is noted that the sharp ellipses considered in this study have a semi-major axis ten times larger than the semi-minor axis as compared with the recent work [3] with the semimajor axis nearly equal to the semi-minor axis. These new models allow the recognition of the different elliptic models from the speckle images as compared with the circular aperture.

\section{Formation of Speckle Images Using Diffusers Modulated by Different Sharp Elliptic Apertures}

In this work, a simple ellipse of semi-major axis ten times the semi-minor axis is investigated. We assume that this sharp ellipse is represented as follows:

$$
f(x, y)=1 ; \quad \frac{x^{2}}{a^{2}}+\frac{y^{2}}{b^{2}} \leq 1 \text { with } b=0.1 a
$$

The numerical ellipse is made from pixels of dimensions $1024 \times 1024$ and is represented as follows:

$$
f(m \Delta x, n \Delta y)=1 ;(m, n)=(1024,1024)
$$

$\Delta x$ and $\Delta y$ are unity.

A diffuser of random phase changes, has the same dimensions like the above described elliptical aperture, is written as follows:

$$
d(x, y)=\exp \left[j\left(\frac{k}{f}\right) r \operatorname{and}(x, y)\right] ; \quad k=\frac{2 \pi}{\lambda}
$$

In matrix form, the simulated diffuser becomes:

$$
d(m \Delta x, n \Delta y)=\exp \left[j\left(\frac{k}{f}\right) \operatorname{rand}(m \Delta x, n \Delta y)\right]
$$

Consider coherent imaging system, with laser uniform illumination incident upon the object (the diffuser) followed by the simulated sharp elliptic aperture. In this case, we can write the complex amplitude transmittance as follows:

$$
c(m \Delta x, n \Delta y)=f(m \Delta x, n \Delta y) \cdot d(m \Delta x, n \Delta y)
$$

Hence, the Fourier transform of Equation (4), realized by a converging lens, gives in its front focal plane this complex amplitude:

$$
\stackrel{v}{c}=\stackrel{f}{f}\left(\frac{m}{\Delta x}, \frac{n}{\Delta y}\right) * \stackrel{v}{d}\left(\frac{m}{\Delta x}, \frac{n}{\Delta y}\right)
$$

where $\stackrel{\vee}{f}=F \cdot T \cdot[f(m \Delta x, n \Delta y)]$,

$$
\stackrel{v}{d}=F \cdot T \cdot[\mathrm{d}(m \Delta x, n \Delta y)] \text {, and } \stackrel{\vee}{c}=F \cdot T[c(m \Delta x, n \Delta y)] \text {. }
$$

The symbol $(*)$ stands for the convolution product.

Equation (5) means that the modulated elongated speckles are formed from the convolution product of both of the Fourier spectrum of the diffuser and the sharp elongated aperture. The direction of speckle is elongated normal to the semi-major axis of the elliptic aperture.

\subsection{Effect of the Aperture Tilting upon the Elongated Speckles}

Assume that the aperture is tilted making an angle $\alpha$ with the aperture plane $(x, y)$ which is not a simple translation. In this case, the sharp elliptic aperture is decomposed into two components one along the optic axis $z$ and the other lies in the plane $(x, y)$ assumed along y-direction written as follows:

$$
f_{\text {tilted }}=\exp [j k y \cos (\alpha)] f(x, y)
$$

The concept of aperture tilting which is represented as a linear phase shift in the Fourier plane resembles the inclined plane wave which gives a linear shift in its front focal plane. Hence, the coherent light emitted from laser beam is incident upon the tilted aperture described by Equation (6) and the transmitted light is incident upon the diffuser described in the precedent section Equation (3) giving this complex amplitude:

$$
c_{\text {tilted }}(m \Delta x, n \Delta y)=f_{\text {tilted }}(m \Delta x, n \Delta y) \cdot d(m \Delta x, n \Delta y)
$$

Substitute from Equation (6) in Equation (7), we rewrite Equation (7) as follows:

$$
\begin{aligned}
& c_{\text {titted }}(m \Delta x, n \Delta y)= \\
& \exp [j k m y \cos (\alpha)] f(m \Delta x, n \Delta y) d(m \Delta x, n \Delta y)
\end{aligned}
$$

Applying the Fourier transform operation and making use of convolution operation we get:

$$
\begin{aligned}
& \stackrel{v}{c}(\text { tilt.apert. })= \\
& \stackrel{v}{f}\left(\frac{m}{\Delta x}, \frac{n}{\Delta y}\right) * v\left(\frac{m}{\Delta x}, \frac{n}{\Delta y}\right) * \delta\left(\frac{m}{\Delta x}, \frac{n-f \cos \alpha}{\Delta y}\right)
\end{aligned}
$$

$\delta$ represents the Dirac-Delta distribution.

This Delta function displaces the whole speckle pattern by an amount equal to $f \cos (\alpha)$ in the direction conjugate to the y direction since $f$ is the focal length of the Fourier transform lens. Hence, for the tilted aperture the complex amplitude of the speckle pattern is written as follows:

$$
\stackrel{v}{c} \text { (tilt.apert. })=\stackrel{v}{c}\left(\frac{m}{\Delta x}, \frac{n-f \cos \alpha}{\Delta y}\right)
$$


It is noted that the linear shift of the whole speckle images is large as compared with the field of view of the whole image. To detect the shift this condition must be fulfilled: $f \cos (\alpha)<3 \sigma ; \sigma$ is the average speckle size.

\subsection{Autocorrelation Algorithm for Speckle Size Evaluation}

The average speckle size of a speckle image is estimated by calculating the auto-covariance function of the digitized intensity speckle pattern as follows:

If $I\left(x_{1}, y_{1}\right)$ and $I\left(x_{2}, y_{2}\right)$ represent the intensities of two points in the imaging plane $(x, y)$, the intensity autocorrelation function is defined by equation

$$
c_{I}(\delta x, \delta y)=\left\langle I\left(x_{1}, y_{1}\right) I\left(x_{2}, y_{2}\right)\right\rangle
$$

where $\delta x=x_{1}-x_{2}, \delta y=y_{1}-y_{2}$ and \langle\rangle corresponds to a spatial average.

The auto-covariance function corresponds to the normalized autocorrelation function of the intensity which has zero base and its full width at half maximum (FWHM) provides a reasonable measurement of the average width of a speckle [6]. In order to use autocorrelation function method to calculate the average speckle size, it requires sufficient sampling speckles in an image to give a reasonable statistical evaluation.

A Matlab program is used [8] to compute the autocovariance of the speckle image. The calculated autocovariance functions are shown and the FWHM of the calculated function gives the average speckle size of the speckle pattern.

\subsection{The Reconstruction Process and the Autocorrelation of Elliptic Apertures}

Since the complex amplitude of modulated speckle images using the above different apertures is given by Equation (5) as follows:

$$
\begin{aligned}
\stackrel{v}{c}(u, v) & =\stackrel{v}{f}\left(\frac{m}{\Delta x}, \frac{n}{\Delta y}\right) * \vee\left(\frac{m}{d}\left(\frac{n}{\Delta x}, \frac{n}{\Delta y}\right)\right. \\
& =\stackrel{v}{f}(u, v) * \stackrel{v}{d}(u, v)
\end{aligned}
$$

$(u, v)=\left(\frac{m}{\Delta x}, \frac{n}{\Delta y}\right)$ are the reduced coordinates in the Fourier plane of speckles.

The reconstruction of the different apertures obtained by operating the inverse Fourier transform upon Equation (5) to get:

$$
R\left(x^{\prime}, y^{\prime}\right)=F \cdot .^{-1}\{\stackrel{v}{f}(u, v) * d(u, v)\}
$$

$\left(x^{\prime}, y^{\prime}\right)$ are the Cartesian coordinates in the imaging reconstruction plane.
Making use of the properties of Fourier transform and convolution, then we get finally this result:

$$
R\left(x^{\prime}, y^{\prime}\right)=f\left(x^{\prime}, y^{\prime}\right) \cdot d\left(x^{\prime}, y^{\prime}\right)
$$

Hence, in the imaging plane we localize the aperture image affected by a noise originated from the diffuser function.

The autocorrelation function of the different apertures is obtained by operating the Fourier transform upon the intensity of the speckle image as follows.

Firstly we get the speckle intensity as the modulus square of the complex amplitude of the speckle Equation $\left(5^{\prime}\right)$ as follows:

$$
I(u, v)=|c(u, v)|^{2}
$$

Operating the $F . T^{-1}$ over Equation (14), we get the autocorrelation function of the multiplication product as follows:

$$
\begin{aligned}
c\left(x^{\prime}, y^{\prime}\right) & =F . .^{-1}\{I(u, v)\} \\
& =F . T^{-1}\left\{|v(u, v) * \stackrel{v}{d}(u, v)|^{2}\right\} \\
& =\left[f\left(x^{\prime}, y^{\prime}\right) \cdot d\left(x^{\prime}, y^{\prime}\right)\right] *\left[f^{*}\left(x^{\prime}, y^{\prime}\right) \cdot d^{*}\left(x^{\prime}, y^{\prime}\right)\right]
\end{aligned}
$$

\section{Results and Discussion}

A diffuser of randomly phase changes used in the formation of all modulated speckle images is shown in Figure 1.

The diffuser is multiplied by the sharp elliptical aperture of semi-major axis ten times the semi-minor axis as shown in the left of Figure 2. A matrix of dimensions $1024 \times 1024$ pixels for this diffused aperture is considered. The elongated speckle pattern is obtained by operating the FFT algorithm upon the diffused aperture as

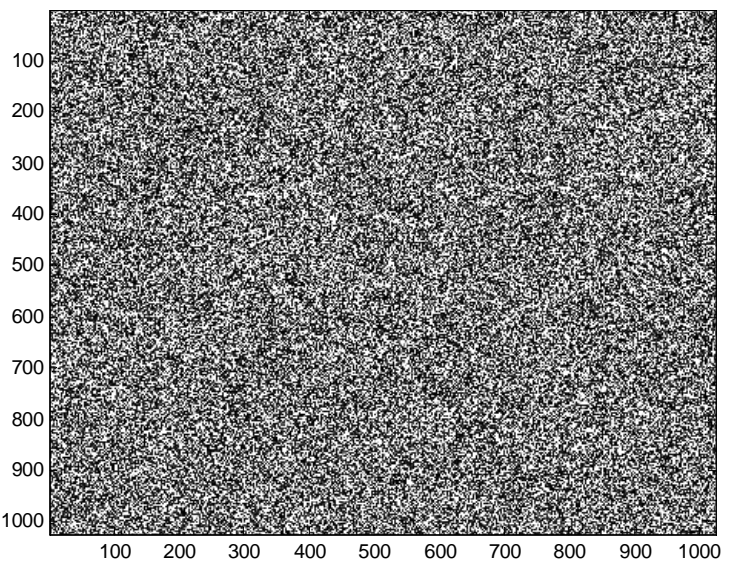

Figure 1. A random diffuser $d(x, y)=\exp [(j 2 \pi / \lambda f) \operatorname{rand}(x, y)]$ with dimensions $1024 \times 1024$ pixels. 

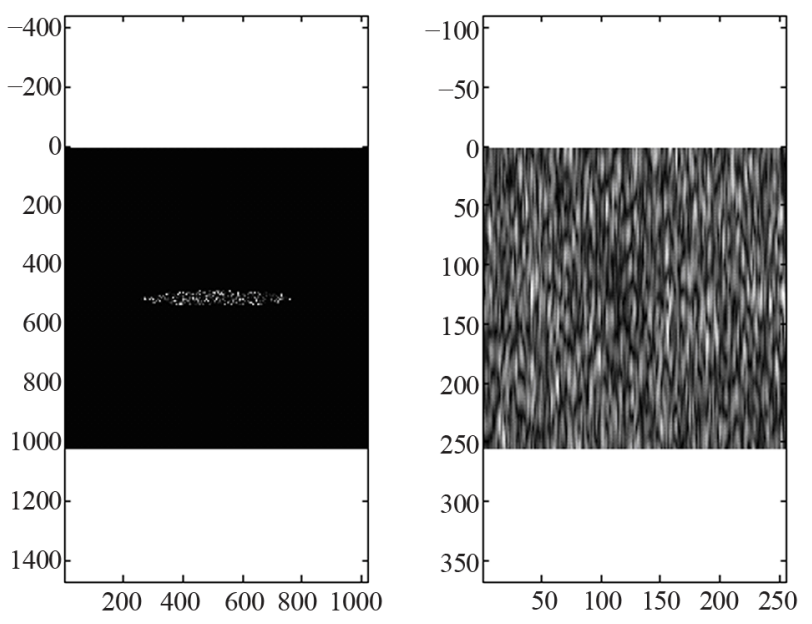

Figure 2. On the left, an elliptical aperture of a semi-major axis $a=2$ and semi-minor axis $b=0.2$ with matrix dimensions of $1024 \times 1024$ pixels is considered. On the right is the corresponding speckle image elongated along the y-direction of matrix dimensions $256 \times 256$ pixels. The elliptical aperture is superimposed over the diffuser and its semimajor axis is extended along $x$-direction.

shown on the right of Figure 2. It is shown that the elongation of speckles is normal to the major axis of the ellipse shown along $\mathrm{x}$-direction giving the elongation directed along the y-axis. In my opinion, this can be interpreted if we consider that the elongated elliptical aperture looks like a slit of finite width along y-direction and hence its Fraunhoffer diffraction pattern located in the Fourier plane is represented by approximate $\operatorname{sinc}(\mathrm{y})$ function varies along $\mathrm{y}$. Consequently, the convolution product of the speckle pattern formed from the ordinary diffuser and the diffraction pattern of the elongated ellipse will give the elongated speckle distribution shown along y-direction as in Figure 2. This reasoning is established from the consideration of sharp elliptic aperture of minor axis equal to the width of the slit along y-direction while the major axis equal to the length of the slit along $\mathrm{x}$-direction. Based, on this analogy all the proposed models are justified.

Figure 3 shows the diffused sharp ellipse with its major axis making an angle $\theta=45^{\circ}$ with the x-axis and the corresponding elongated speckle with the elongation orthogonal to the pupil major axis. It is shown that the direction of the ellipse is recognized from the direction of the elongated speckle where both of them must be orthogonal to each other.

The effect of aperture misalignment is shown in Figure 4. The tilting angle $\alpha$ is the angle between the incident ray and the normal to the aperture plane. This angle is taken to be $3^{\circ}$ and $4^{\circ}$ as shown in figures (b), (c) and compared with the case of perfectly aligned aperture shown in Figure 4(a). This tilting is completely different as compared with aperture orientation in its plane (results
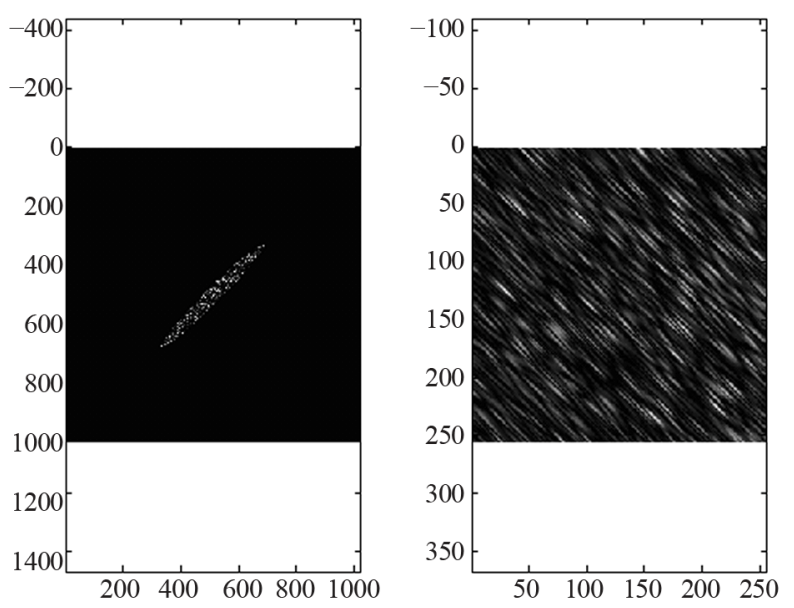

Figure 3. On the left, an elliptical aperture making an angle of $45^{\circ}$ with the $x$-axis and of matrix dimensions of $1024 \times$ 1024 pixels is shown. On the right is the corresponding speckle image elongated normal to the semi-major axis of matrix dimensions $256 \times 256$ pixels. The elliptical aperture is superimposed over the diffuser.

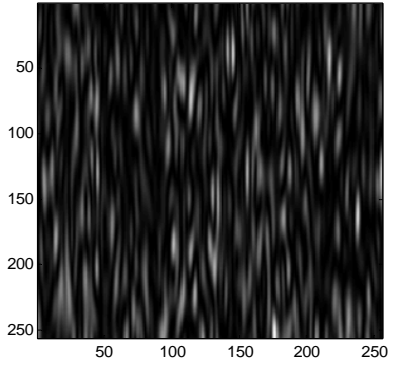

(a)

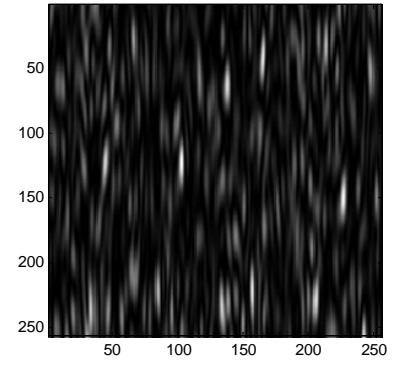

(b)

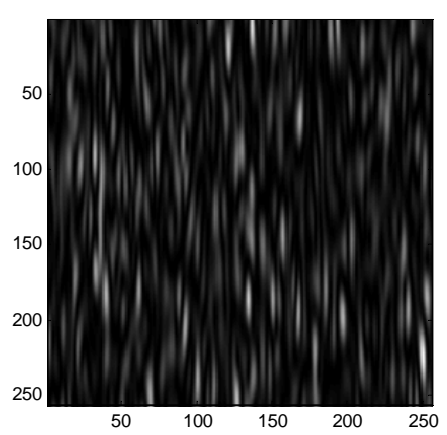

(c)

Figure 4. Three different elongated speckle images using the sharp elliptic aperture shown in Figure 2. (a) On the left is the elongated speckle for aligned sharp elliptic aperture. While (b) and (c) are obtained for misaligned aperture taking the tilting angle $\alpha=3^{\circ}$ and $4^{\circ}$ respectively.

shown in Figures 1 and 2). If we compare the three elongated speckle images taking the aligned aperture as a reference hence we can predict any misalignment due to the aperture tilting by examining the speckle pattern. Any larger inclinations will give different speckle patterns since the inclination in the pupil plane is transformed into 
linear shift in the Fourier plane. Hence, larger aperture tilting give a difference as compared with the aligned aperture but small tilting is interesting to check the alignment of the optical system.

Also, larger aperture tilting give a difference as compared with the aligned aperture but small tilting is interesting to check the alignment of the optical system.

The plus symbol pupil is composed of two orthogonal ellipses modulated by the diffuser is shown in the left of Figure 5 for a matrix of $2048 \times 2048$ pixels. On the right is the corresponding speckle image. The elongation of the speckle image is shown directed along both of the $x$ and $y$-axes. Also, another arrangement of a snow flake or four ellipses superimposed over the diffuser and the corresponding elongated speckle image is plotted as in Figure 6. This arrangement shows a complicated elongation since each ellipse gives speckle elongation orthogonal to its elliptical major axis. Hence, the recognition of the snow flake pupil is attributed to the four different directions shown for the elongation of the same speckle.

All the elongated speckle images shown in Figures 26 are of dimensions $256 \times 256$ pixels.

The 3rd model of a pupil in the form of an airplane superimposed over the diffuser of matrix dimensions 2048 $\times 2048$ pixels is numerically constructed as shown in Figure 7. The corresponding speckle image is obtained by operating the FFT showed a specific elongation along the plane $(x, y)$ represented as in Figure 8.

The profile shape of the autocorrelation intensity is shown as in Figure 9(a), along the $x$-axis corresponding to the elongated speckles Figure (8) for the airplane pupilof $2048 \times 2048$ pixels. Also, the autocorrelation profile along the $y$-axisis shown in Figure 9(b). The spec-
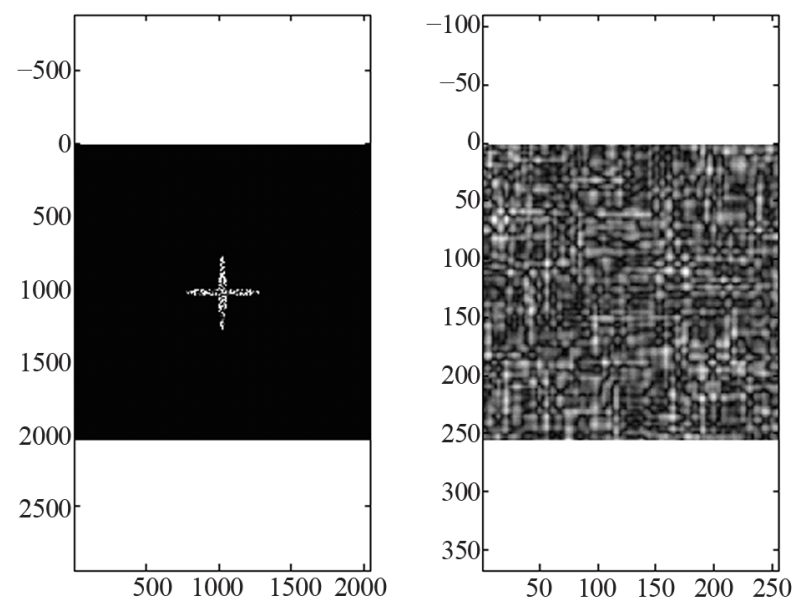

Figure 5. On the left, a snow flake pupil of two orthogonal ellipses of matrix dimensions $2048 \times 2048$ pixels is shown. On the right is the corresponding speckle image elongated along the $x$ - and $y$-directions and the matrix dimensions are $256 \times 256$ pixels. The elliptical aperture is superimposed over the diffuser.
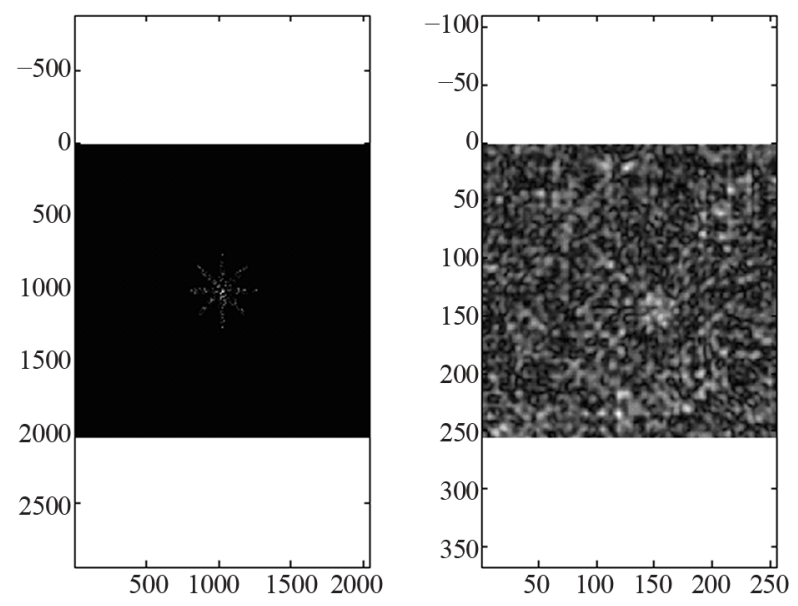

Figure 6. On the left, a snow flake pupil superimposed over the diffuser of matrix dimension $2048 \times 2048$ pixels is shown. On the right is the corresponding speckle image elongated along the different directions of matrix dimensions $256 \times 256$ pixels.

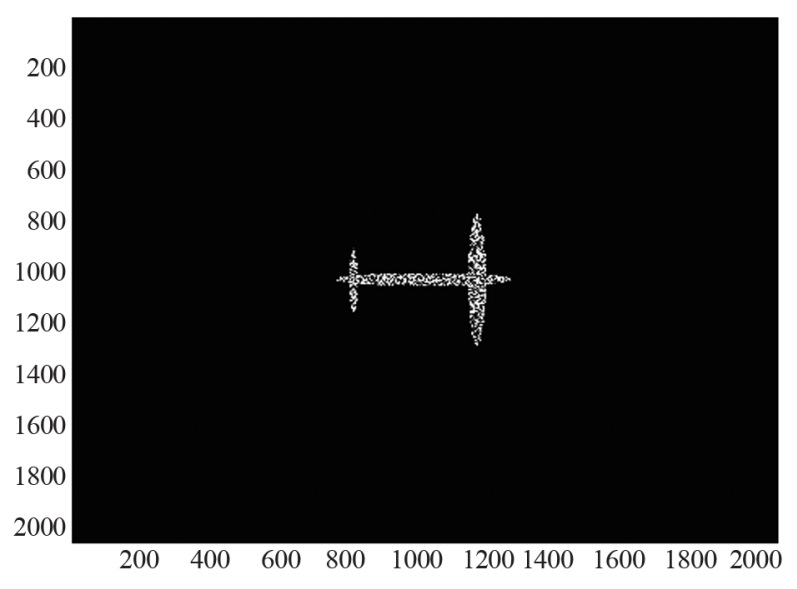

Figure 7. A pupil in the form of an airplane superimposed over the diffuser of matrix dimensions $2048 \times 2048$ pixels.

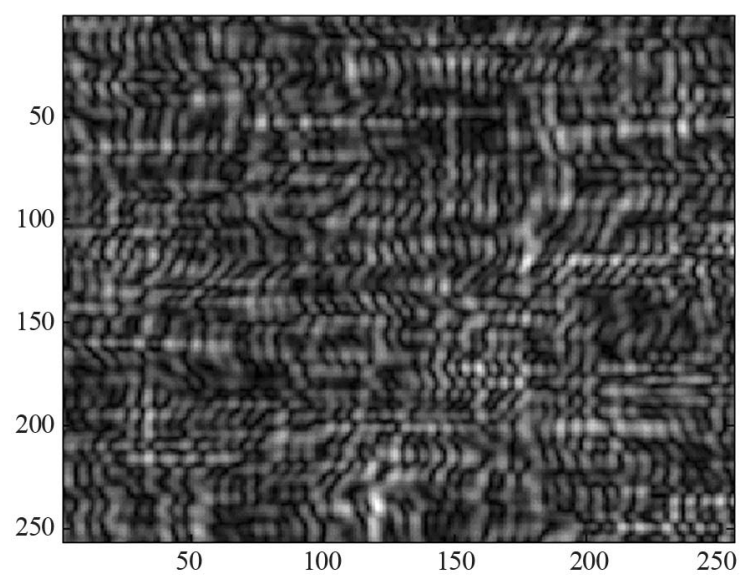

Figure 8. Speckle elongation in different directions of dimensions $256 \times 256$ pixels corresponding to the airplane diffused pupil of dimensions $2048 \times 2048$ pixels. 


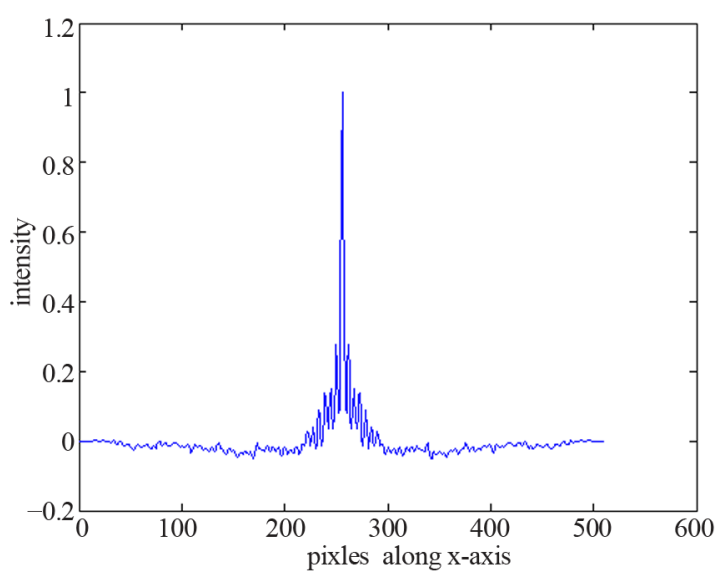

(a)

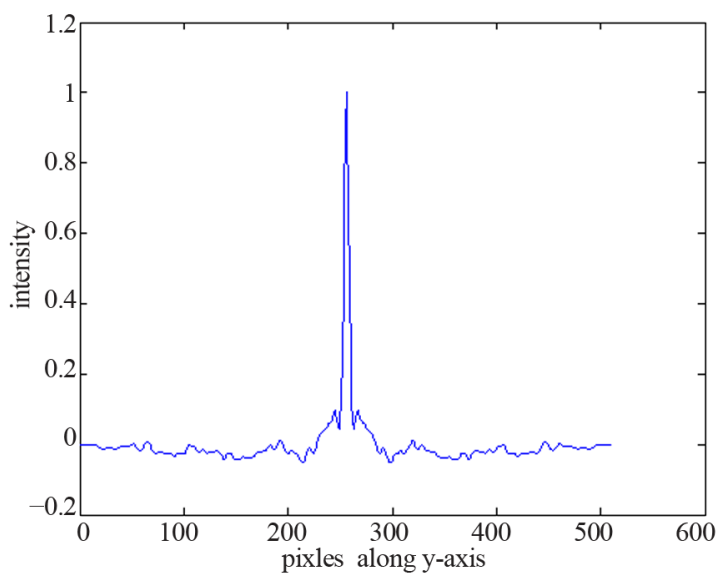

(b)

Figure 9. (a) Autocorrelation intensity along the $\mathbf{x}-$ axis of the elongated speckles shown in Figure (8) for the airplane pupil of $2048 \times 2048$ pixels. The average speckle size is $\sigma_{\mathrm{x}}=$ ( $4.5 \mathrm{~mm} / 512$ pixels) $(5$ pixels $)=44 \mathrm{~mm}$; (b) Autocorrelation intensity along the y-axis of the elongated speckles shown in Figure (8) for the airplane pupil of $2048 \times 2048$ pixels. The average speckle size is $\sigma_{\mathrm{y}}=(3.6 \mathrm{~mm} / 512$ pixels $)(8$ pixels $)=$ $56 \mathrm{~mm}$.

kle size is computed by taking the full width at half maximum along $x$-direction, referring to Figure 9(a) as follows: $\mathrm{FWHM}=\Delta x=5$ pixels and along the y-direction, referring to Figure 9(b) as: $\Delta y=8$ pixels. Hence the average speckle size along $x$-direction is calculated, assuming field of view in the speckle image equal $4.5 \mathrm{~mm}$ $\times 3.6 \mathrm{~mm}$, as follows:

$$
\sigma_{x}=(4.5 \mathrm{~mm} / 512 \text { pixels })(5 \text { pixels })=44 \mathrm{~mm}
$$

while the average speckle size along the y-direction is computed as:

$$
\sigma_{y}=(3.6 \mathrm{~mm} / 512 \text { pixels })(8 \text { pixels })=56 \mathrm{~mm}
$$

For comparison, the average speckle size for circular aperture of radius 128 pixels is obtained (referring to Figures 10-12 as follows):

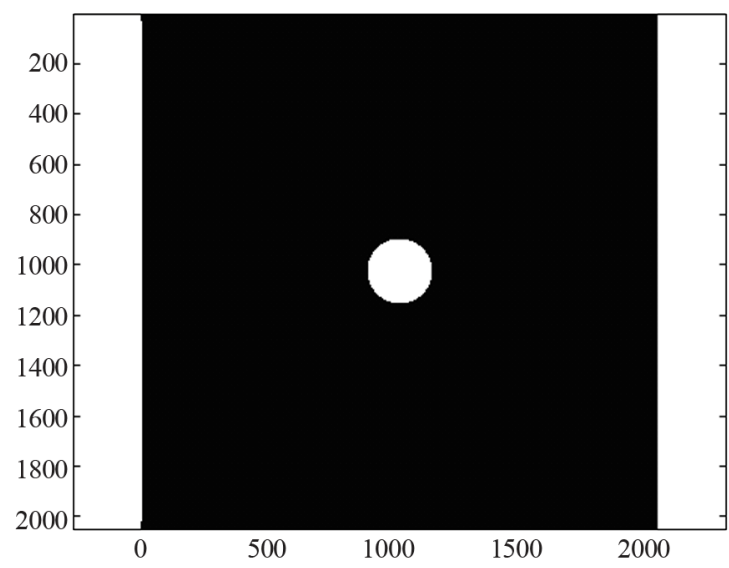

Figure 10. uniform circular aperture of pixels dimensions $2048 \times 2048$ and radius 128 pixels.

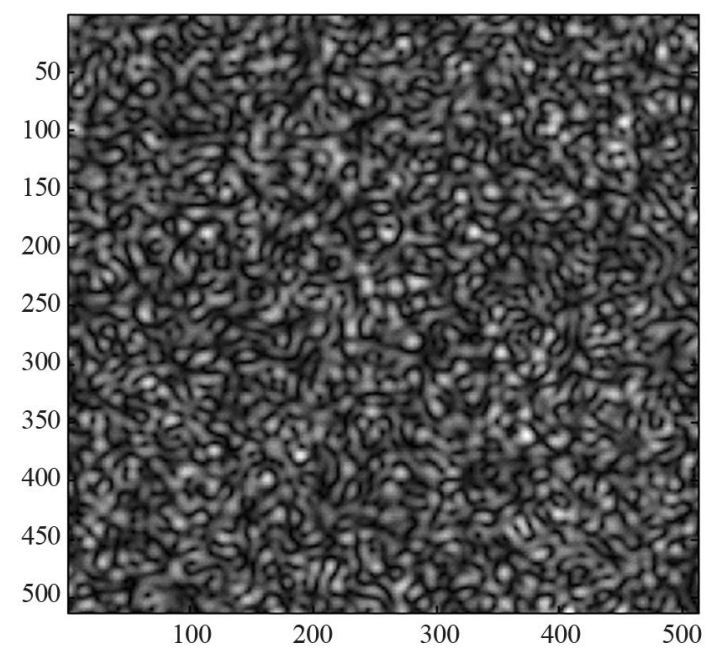

Figure 11. The field of view is $4.5 \mathrm{~mm} \times 3.6 \mathrm{~mm}$ corresponding to $512 \times 512$ pixels for the speckle image, and is obtained for the diffuser provided with uniform circular aperture shown in Figure 10.

$$
\begin{aligned}
& \sigma_{x}=(4.5 \mathrm{~mm} / 2048 \text { pixels })(10 \text { pixels })=44 \mu \mathrm{m} \\
& \sigma_{y}=(3.6 \mathrm{~mm} / 2048 \text { pixels })(10 \text { pixels })=35 \mu \mathrm{m}
\end{aligned}
$$

Another set of three different speckle images using a circular aperture are plotted as in Figure (13). On the left is the speckle for the aligned circular aperture. While the other two figures (b), (c) are obtained for misaligned circular apertures, taking the tilting angles $\alpha=3^{\circ}$ and $4^{\circ}$ respectively. All apertures have equal radii of 64 pixels. The autocorrelation intensity of the corresponding speckle patterns are shown as in Figures 14(a)-(c). It is shown that the aligned aperture is recognized from its speckle pattern Figure 14(a) since it is different from the tilted aperture Figures 14(b) and (c). Hence, any misalignment due to aperture tilting is recognized referring to its different speckle pattern. 


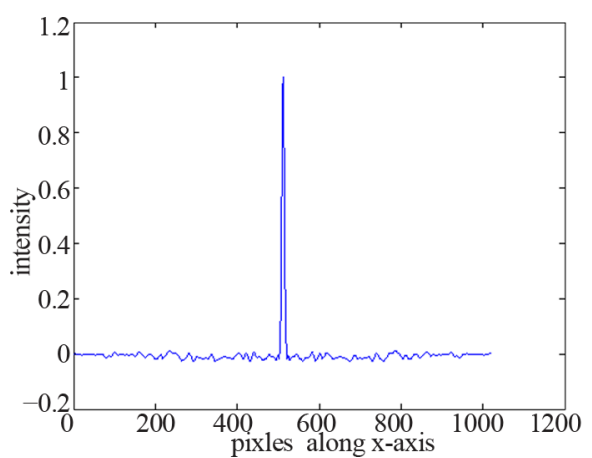

(a)

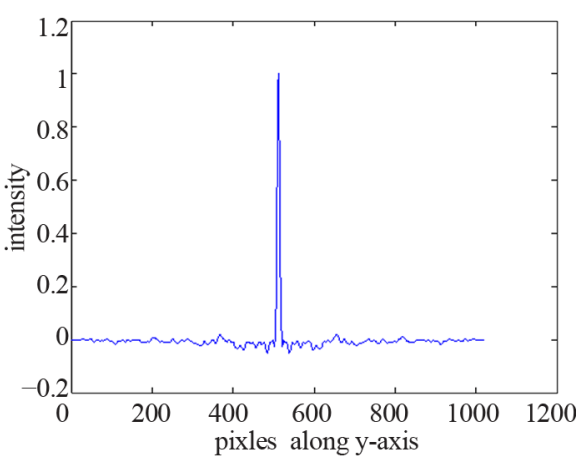

(b)

Figure 12. (a) Autocorrelation intensity of the speckle image shown in Figure 10 along the $x$-axis. The FWHM of the speckle size corresponding to the 10 pixels shown on the autocorrelation peak is equal to: $s=(4.5 \mathrm{~mm} / 1024$ pixels $)(10$ pixels $)=44$ $\mathrm{mm}$;(b) Autocorrelation intensity of the speckle image shown in Figure 9 along $y$-axis. The FWHM of the speckle size corresponding to the 10 pixels shown on the autocorrelation peak is equal to: $\mathrm{s}=(3.6 \mathrm{~mm} / 1024$ pixels $)(10$ pixels $)=35 \mathrm{~mm}$.

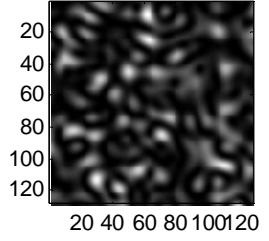

(a)

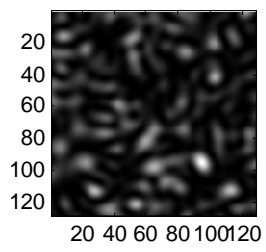

(b)

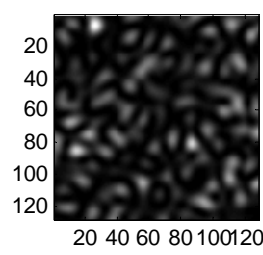

(c)

Figure 13. Three different speckle images using a circular aperture. On the left is the speckle for the aligned circular aperture. While the other two figures (b), (c) are obtained for misaligned circular apertures, taking the tilting angles $\alpha=3^{\circ}$ and $4^{\circ}$ respectively. All apertures have equal radii of 64 pixels.

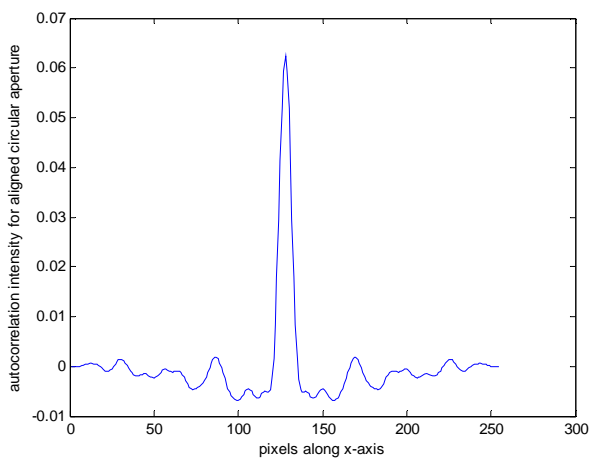

(a)

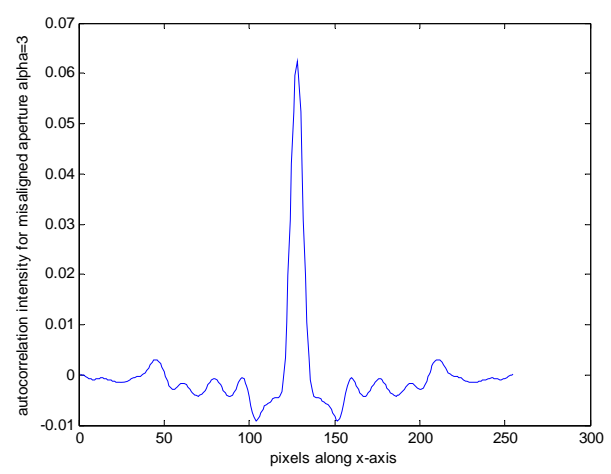

(b)

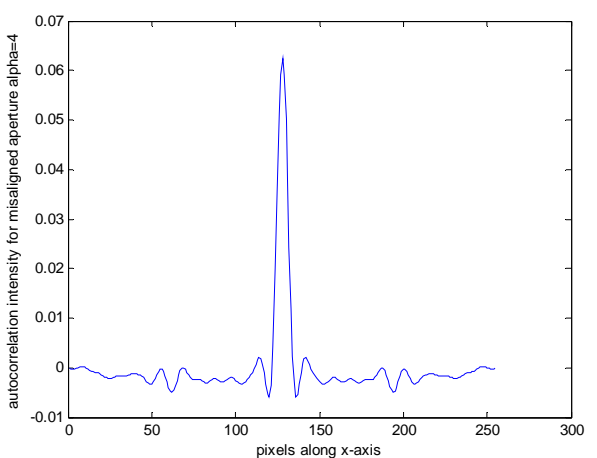

(c)

Figure 14. (a) The autocorrelation intensity of the speckle pattern corresponding to the aligned circular aperture. (b) The autocorrelation intensity of the speckle pattern corresponding to misaligned circular aperture of tilting angle $\alpha=3^{\circ}$. (c) The autocorrelation intensity of the speckle pattern corresponding to misaligned circular aperture of tilting angle $\alpha=4^{\circ}$. 
The reconstruction of the two crossed ellipses using the inverse Fourier transform of matrix dimensions 2048 $\times 2048$ pixels is shown as in Figure 15. Also, the reconstructed image of the aperture of four crossed ellipses is shown as in Figure 16.

The autocorrelation of the two orthogonal ellipses obtained from the reconstruction of the speckle intensity is shown as in Figure 17 and the autocorrelation of the four equally spaced ellipses where the angle between each two is $\theta=45^{\circ}$ are shown as in Figure 18. The autocorrelation images shown in Figures $\mathbf{1 7}$ and $\mathbf{1 8}$ are obtained using Equation (15).

\section{Conclusions}

The motivation of the proposed sharp models is for the sake of its recognition by comparing the different speckle images with the ordinary speckle image for uniform circular aperture and to check its alignment in its plane.

The snowflake and the airplane models showed a relatively complicate elongation since each part give elonga-

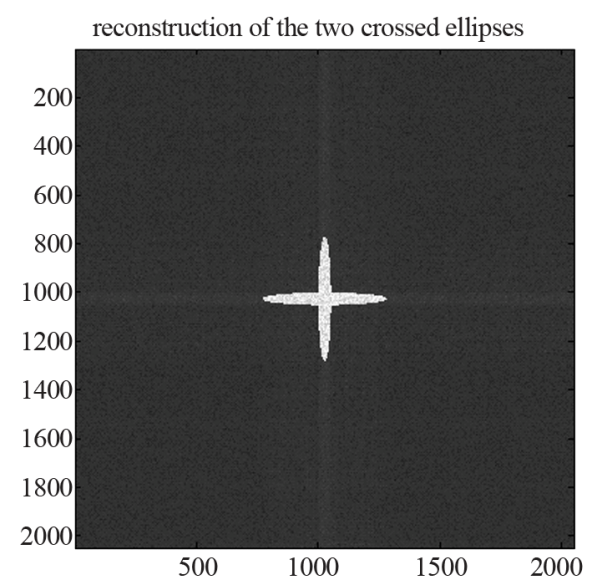

Figure 15. Reconstruction of the plus symbol pupil of matrix $2048 \times 2048$ pixels.

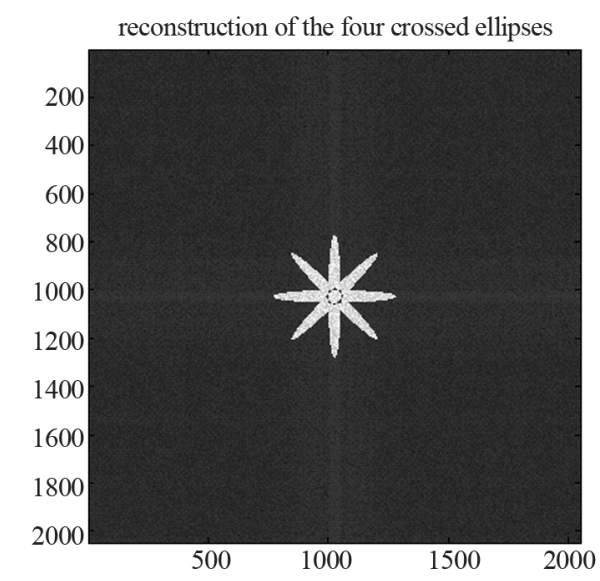

Figure 16. Reconstruction of the snow flake pupil of matrix $2048 \times 2048$ pixels.

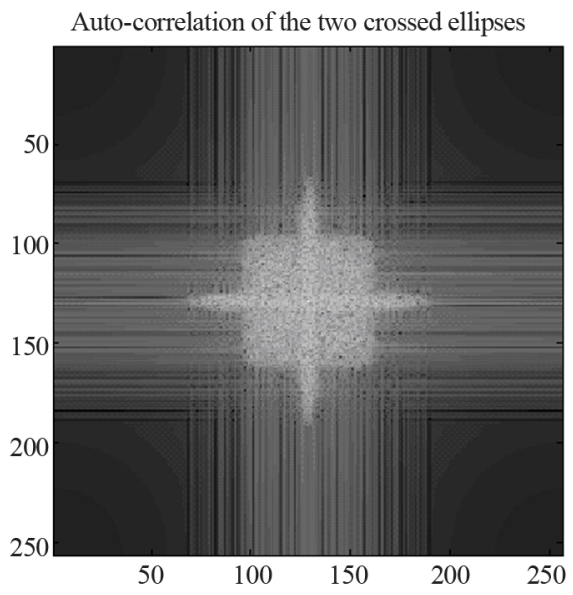

Figure 17. The autocorrelation of plus symbol pupil or the two orthogonal ellipses.

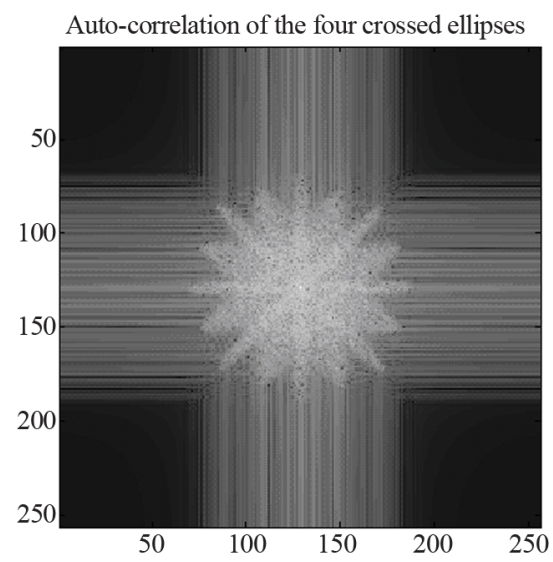

Figure 18. The autocorrelation of the snow flake pupil or the four crossed ellipses.

tion normal to the concerned elliptical part allowing again its recognition.

The alignment of optical microscopic systems can be verified by testing the aperture inclination using speckle techniques.

A potential application of this work may be extended to polychromatic illumination using a mixture of $\mathrm{He}-\mathrm{Ne}$ laser and Ar ion laser in order to differentiate the colored parts of the elliptic apertures.

\section{REFERENCES}

[1] G. G. Mu, Z. Q. Wang, Q. Gong, Q. W. Song and F. X. $\mathrm{Wu}$, "White-Light Image Processing Using Oriented Speckle-Screen Encoding," Optics Letters, Vol. 10, No. 8, 1985, pp. 375-377. doi:10.1364/OL.10.000375

[2] N. Barakat, A. M. Hamed, H. El Ghandoor, K. El-Dohimy, M. A. Fadly and O. A. Abdel Ghafar, "A Photographic Encoder Applied to an Optical Processor Using Speckle Techniques," Journal of Modern Optics, Vol. 38, No. 1, 1991, pp. 203-208. 
doi:10.1080/09500349114550221

[3] A. M. Hamed, "Discrimination between Speckle Images Using Deformed Apertures," Optical Engineering, Vol. 56, No. 1, 2011, pp. 1-7.

[4] P. Lehmann, S. Patzelt and A. Schöne, "Surface Roughness Measurement by Means of Polychromatic Speckle Elongation," Applied Optics, Vol. 36, No. 10, 1997, pp. 2188-2197. doi:10.1364/AO.36.002188

[5] P. Lehmann, "Aspect Ratio of Elongated Polychromatic Far-Field Speckles of Continuous and Discrete Spectral Distribution with Respect to Surface Roughness Characterization," Applied Optics, Vol. 41, No. 10, 2002, pp. 20082014. doi:10.1364/AO.41.002008

[6] Z. H. Yuan, et al., "Comparison of Surface Roughness Measurement in Dichromatic Speckle Patterns with Autocorrelation Method," Advanced Materials Research, Vol. 189, 2011, pp. 680-683.

[7] A. S. Kumar and R. M. Vasu, "Imaging with Oriented Photographic Diffusers," Instrumentation and Services Unit, Indian Institute of Science, Bangalore, 2002.

[8] H. Lin, "Speckle Mechanism in Holographic Optical Coherence Imaging," Ph.D. Dissertation, University of Missouri, Kansas City, 2009, pp. 57-58.

[9] H. Lin and P. Yu, "Speckle Mechanism in Holographic Optical Imaging," Optics Express, Vol. 15, No. 25, 2007, pp. 16322-16327. doi:10.1364/OE.15.016322

[10] J. W. Goodmann, "Statistical Properties of Laser Speckle
Patterns in Laser Speckle and Related Phenomena," Springer-Verlag, New York, 1984.

[11] Y. Piederriere, J. Le Meur, J. Cariou, J. Abgrall and M. Blouch, "Particle Aggregation Monitoring by Speckle Size Measurement, Application to Blood Platelets Aggregation," Optics Express, Vol. 12, No. 19, 2004, pp. 45964601. doi:10.1364/OPEX.12.004596

[12] P. Lehmann, "Surface Roughness Measurement Based on the Intensity Correlation Function of Scattered Light under Speckle Pattern Illumination," Applied Optics, Vol. 38, No. 7, 1999, pp. 1144-1152. doi:10.1364/AO.38.001144

[13] R. Berlasso, F. Perez Quintian, A. M. Rebollo, A. C. Raffo and G. N. Gaggioli, "Study of Speckle Size of Light Scattered from Cylindrical Rough Surfaces," Applied Optics, Vol. 39, No. 31 , 2000, pp.5811-5819. doi:10.1364/AO.39.005811

[14] L. T. Alexander, E. J. Harvey and R. A. Weeks, “Average Speckle Size as a Function of Intensity Threshold Level: Comparison of Experimental Measurements with Theory," Applied Optics, Vol. 33, No. 35, 1994, pp. 82408250. doi:10.1364/AO.33.008240

[15] K. Matsushima, H. Schimmel and F. Wyrowski, "Fast Calculation Method for Optical Diffraction on Tilted Planes by Use of Angular Spectrum of Plane Waves," Journal of the Optical Society of America, Vol. 20, No. 9, 2003, pp. 1755-1762. doi:10.1364/JOSAA.20.001755 